\title{
VALORES ÉTICOS Y FORMACIÓN CURRICULAR EN ODONTOLOGÍA
}

\author{
Luis A. Vicentela ${ }^{1}$, Carmen Gloria Narváez ${ }^{1}$, Manuel Velásquez ${ }^{1}$
}

Resumen: Para identificar los valores éticos de los estudiantes de odontología y relacionarlos con el perfil de egreso, se elaboró una encuesta, aplicada a 1.281 estudiantes de ocho sedes que imparten odontología en Chile. Esta contenía 24 valores y cinco categorías de relevancia, estimando un promedio general por curso y género. En orden de relevancia, valores como felicidad, responsabilidad, sinceridad, respeto y bienestar fueron los más destacados; mientras que innovación, liderazgo, civismo, participación y reconocimiento los menos resaltados. En los primeros, no hay diferencias significativas por curso pero sí por género $(p<0,05)$, excepto en bienestar, mientras que en los menos destacados no hay diferencias significativas por género, pero sí por curso $(\mathrm{p}<0,05)$. Del análisis de los perfiles destacan los valores de liderazgo, participación, cooperación y convivencia. Sin embargo, estos se encuentran al final de la escala de preferencia de los alumnos. De los cinco más valorados por los estudiantes, solo responsabilidad y respeto destacan en uno de los perfiles de egreso. En conclusión, los perfiles de egreso profesional deben incorporar valores que fortalezcan el desarrollo ético y tengan en cuenta la impronta valórica de los alumnos, de forma de alinearlos con los definidos por la institución y el propio perfil.

Palabras clave: ética, valores, perfil profesional, odontología, curriculum

\section{Ethical values and curricula training in Dentistry}

\begin{abstract}
In order to identify ethical values of Dentistry students related to the profile of career ending, a survey was elaborated and applied to 1281 students of 8 sites teaching Dentistry in Chile. This contains 24 values and five relevant categories, estimating a general average by course and gender. In order of relevance, values such as happiness, responsibility, sincerity, respect and wellbeing were most highlighted; while innovation, leadership, civism, participation and acknowledgement were those less highlighted. In the first ones there were no significant differences by course but yes by gender $(\mathrm{p}<0,05)$, except wellbeing, while in those less highlighted, there were no significant differences by gender but yes by course $(\mathrm{p}<0,05)$. Profile analysis emphasizes the values of leadership, participation, cooperation and coexistence. Nevertheless, those are found at the end of preference scale of students. Of the five most valued by students, only responsibility and respect were highlighted in one profile of career ending. In conclusion, the profile of professional career ending must incorporate values to strengthen ethical development, taking into account the values highlighted by students, conforming them to those defined by the institution and personal profiles.
\end{abstract}

Key words: ethics, values, professional profile, dentistry, curriculum

\section{Valores éticos e formaçáo curricular em odontologia}

Resumo: Para identificar os valores éticos dos estudantes de odontologia e relacioná-los com o perfil do egresso, se elaborou uma enquete aplicada a 1.281 estudantes de oito sedes que oferecem odontologia no Chile. A enquete continha 24 valores e cinco categorias de relevância, estimando uma média geral por curso e gênero. Em ordem de relevância, valores como felicidade, responsabilidade, sinceridade, respeito e bem-estar foram os mais destacados; enquanto que inovação, liderança, civismo, participação e reconhecimento os menos ressaltados. Nos primeiros, não há diferenças significativas por curso, porém sim por gênero $(\mathrm{p}<0,05)$, exceto em bem-estar, enquanto que nos menos destacados, não há diferenças significativas por gênero, porém sim por curso $(\mathrm{p}<0,05)$. Da análise dos perfis destacam os valores de liderança, participação, cooperação e convivência. No entanto, estes se encontram ao final da escala de preferência dos alunos. Dos cinco mais valorados pelos estudantes, só responsabilidade e respeito se destacam em um dos perfis do egresso. Em conclusão, os perfis do egresso profissional devem incorporar valores que fortaleçam o desenvolvimento ético e tenham em conta o perfil valórico dos alunos, de forma a alinhá -los com os definidos pela instituição e o próprio perfil.

Palavras-chave: ética, valores, perfil profissional, odontologia, curriculum

\footnotetext{
${ }^{1}$ Facultad de Odontología Universidad del Desarrollo, Concepción, Chile Correspondencia: lvicentela@udd.cl
} 


\section{Introducción}

La formación valórica en odontología se ha difundido como un componente necesario en el perfil del profesional, por su directa vinculación con personas en la práctica diaria y por su responsabilidad social, como carrera al servicio del bienestar de la comunidad. Dichos valores, si bien son generados en la familia, inculcados en la educación básica y moldeados en la relación social, el periodo de vida universitaria constituye una oportunidad de complementación y refuerzo para el comportamiento durante la vida profesional. La educación profesional en odontología existe para formar buenos dentistas, bien provistos y dispuestos a mejorar la situación de salud bucal de la población(1), idea ya planteada por Bridgman y otros (1999)(2), quienes indican que la formación de buenos dentistas implica realzar y promover la salud general y bucodentaria, además de proporcionar bienestar a la gente que atienden, de manera justa, y respetando su dignidad, autonomía y derechos. En otras palabras, la buena odontología depende de individuos comprometidos a servir a la sociedad y sus pacientes en forma justa, es decir éticamente(1).

Por otro lado, también se ha señalado que esta profesión implica espíritu de servicio. Se asume que, al adquirir el derecho de ejercer esta profesión, el deber es hacerlo siempre bien(3). En este contexto, una de las justificaciones para incorporar valores éticos como cuerpo de contenidos en odontología es facilitar el desarrollo personal y profesional de los aspirantes a dentistas y aportar al desarrollo de personas más sensibles a los valores humanos universales(4).

Desde una perspectiva conceptual, los valores existen por las relaciones sociales, por la sociedad y por el hombre, y están influenciados por factores tales como la educación familiar, las tradiciones, la idiosincrasia, la educación formal y las ideas y creencias, entre otros(5). No obstante, se ha planteado el debate sobre si es necesario incluir aspectos valóricos en los proyectos curriculares de odontología, instalados en asignaturas como ética y bioética. Lo anterior radica en que aún no está del todo claro si la bioética es o no un conocimiento indispensable para el agente de salud(5). En el extremo de esta tensión, se ha señalado que todo problema clínico encierra un dilema ético(6) y esto sugiere que todo acto sanitario contiene a su vez un aspecto valórico que atender y conocer(7). Sin embargo, esto se plantea desde el ejercicio profesional clínico, asumiendo una formación previa que fortalece estos aspectos valóricos.

Desde un punto de vista curricular, se ha señalado que los valores son contenidos, explícitos o implícitos, inevitables en la educación(4), es decir, cualquier actividad educativa estaría condicionada por los valores. También es sabido que los elementos del curriculum (selección de contenidos, diseño de metodologías, estrategias de evaluación, etc.) están influidos por los valores de las personas que participan de él(7).

Una aproximación a la forma como los alumnos perciben y representan los valores es una tarea de largo plazo, que implica a lo menos el seguimiento de una cohorte, de manera que se permita conocer cómo se vivencian estos valores en el aula y cómo se proyectan en el ejercicio profesional. Este estudio es un punto de partida que se propone analizar las percepciones declaradas por los alumnos y establecer tanto la relevancia que los distintos grupos dan a los valores éticos y morales en el contexto de la carrera de odontología, como las discrepancias que subyacen entre los grupos estudiados y entre estos y los valores declarados en el perfil y contenidos curriculares de la carrera.

\section{Método}

El instrumento utilizado para la recogida de datos se basó en los trabajos de Capote y otros(8) y García y otros(9). Se seleccionó la encuesta de García y otros(9) como base del instrumento definitivo, al que se incorporó explicaciones y formato de acuerdo con la idiosincrasia de los encuestados (área odontológica). La encuesta quedó conformada por 24 ítems con cinco categorías de respuesta e instrucciones para la aplicación. La validación del instrumento se realizó utilizando el criterio de juicio de expertos, seleccionados de acuerdo con el coeficiente K de competencia(10). La evaluación de expertos consideró adecuación contextual del instrumento, parsimonia y coherencia que, en conjunto, definen la validez de contenido (grado en que la muestra de ítemes que componen el instrumento es representativa del universo o do- 
minio al que el instrumento está referido; es una validez fundamentalmente teórica o de representación)(11). Considerando las observaciones del juicio de expertos, se diseñó el instrumento final con modificaciones menores que no afectaron el número de ítems ni de categorías. Se realizó un piloto a una muestra de alumnos seleccionados, aplicándose al $10 \%$ de la población de alumnos de $1^{\text {er }}$ año y al $10 \%$ de alumnos de $5^{\circ}$ año de la carrera de odontología de la Universidad del Desarrollo, sede Concepción. Este pilotaje permitió verificar si existían observaciones respecto de la validez del instrumento, observándose que era comprensible y coherente, además de susceptible de ser autoadministrado.

Para la aplicación del instrumento se contactó a los decanos de odontología de universidades participantes de la Asociación Chilena de Enseñanza de la Odontología (ACHEO) y se informó de la ejecución de este proyecto comprometiendo participación. Se procedió luego a encuestar a los estudiantes que consintieron en participar, insistiendo en el carácter anónimo de la información que se obtendría y se agradeció la colaboración.

Para analizar los perfiles de egreso de las carreras de odontología de las distintas sedes y determinar la presencia de valores en su descripción, se revisó cada uno de ellos de acuerdo con su declaración en sitio web y se categorizó la presencia de términos asociados a los valores encuestados en los alumnos. Con ello se estableció si existía coherencia entre el valor declarado en el perfil y la relevancia que el propio alumno le da a ese mismo valor.

\section{Resultados}

La muestra de esta investigación correspondió a 1.281 estudiantes de odontología distribuidos de la siguiente forma: 730 estudiantes de primer año y 551 estudiantes de quinto año, pertenecientes a 8 sedes que imparten la carrera de odontología en universidades chilenas.

La encuesta quedó conformada por 24 ítems (cada ítem un valor) con 5 categorías de respuesta en escala Likert ( $1=$ sin importancia, $2=$ poco importante, $3=$ medianamente importante, $4=\mathrm{im}$ portante, 5=muy importante) e instrucciones para la aplicación.
La jerarquía asignada a los valores encuestados se distribuye de mayor a menor, en función de las medias aritméticas obtenidas, de acuerdo con el gráfico 1 .

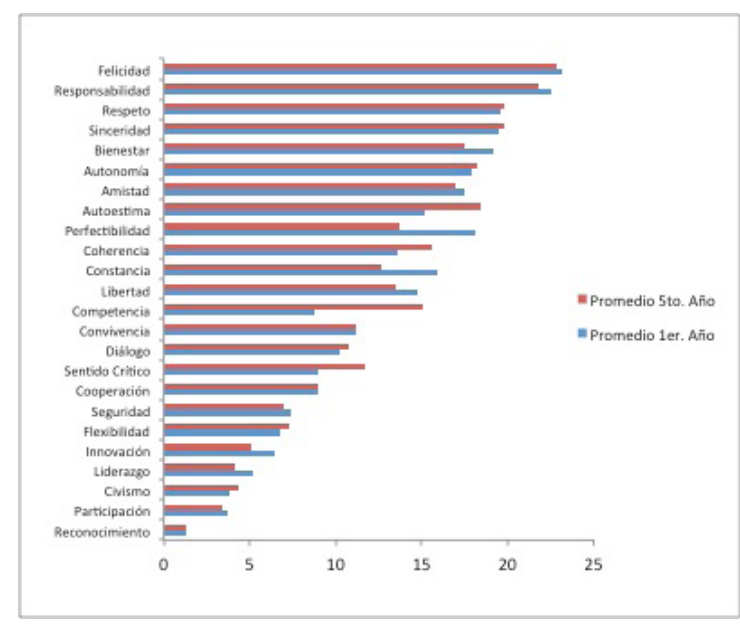

Gráfico $\mathrm{N}^{\circ} 1$ : Distribución jerárquica de los valores encuestados.

Pruebas no paramétricas: Se realizaron pruebas de estadística no paramétrica para los valores jerarquizados en las primeras 5 y en las últimas 5 preferencias. En la prueba de Kruskal Wallis, de los valores correspondientes a las primeras 5 jerarquías, con la variable de agrupación "género", se observa diferencias en los valores felicidad, responsabilidad, respeto y sinceridad $(\mathrm{p}<0.05)$, no existiendo diferencias significativas en el valor bienestar $(p>0.05)$ (tabla 1).

Para los valores jerarquizados en las últimas 5 preferencias, se observa que no hay diferencias significativas en los valores innovación, liderazgo, civismo, participación y reconocimiento $(\mathrm{p}>0.05)$ (variable de agrupación "género") (tabla 2).

En la prueba de Kruskal Wallis, de los valores correspondientes a las primeras 5 jerarquías con la variable de agrupación "curso", se observa que no hay diferencias significativas en los valores felicidad, responsabilidad, respeto, sinceridad y bienestar ( $>>0.05)$ (tabla 3).

Para los valores jerarquizados en las últimas 5 preferencias, se observa diferencias significativas en los valores innovación, liderazgo, civismo, participación y reconocimiento ( $\mathrm{p} \leq 0.05)$ (variable de agrupación "curso") (tabla 4). 
Del análisis de los perfiles de egreso de las universidades encuestadas, se desprende que lo más destacado en ellos son valores de liderazgo, participación, cooperación y convivencia. Sin embargo, la relevancia que los alumnos le otorgan a esos valores, en el caso de los dos primeros, se encuentran al final de la escala de preferencias. Por otro lado, de los cinco términos más valorados por los estudiantes solo responsabilidad y respeto destacan en uno de los perfiles profesionales (tabla 5).

\section{Discusión}

El tema valórico en la formación de profesionales odontólogos al servicio de la comunidad es de extrema relevancia, puesto que constituye la guía del comportamiento individual y social que definirá su desempeño profesional. Por ello, la educación en valores es una herramienta básica para justificar las decisiones morales, en especial porque la tarea del odontólogo se basa en la relación interpersonal y sus decisiones tienen impacto de diversa índole en el bienestar de las personas atendidas.

Por lo anterior, la declaración explícita de los valores a potenciar en el estudiante debe estar reflejada en el perfil de egreso, lo que a su vez debe traducirse en programas y metodologías que incentiven dichos valores. Y para que esa definición sea más acertada, es necesario saber cuáles son los valores que los alumnos, por su formación social y familiar, traen consigo y cuáles cree la institución deben potenciarse para alinear la declaración de intención expresada en el perfil de egreso y los valores que se deba recalcar.

Los resultados de esta investigación demuestran que los valores que efectivamente son declarados en algunos perfiles no se encuentran entre los que más destacan los alumnos, como tampoco se aprecia diferencias entre los alumnos de primero y quinto año, evidenciando que en el proceso formativo tampoco se altera esa cadena de valores, aun cuando la institución lo declara como un objetivo a conseguir en dicho proceso.

De lo anterior se desprenden dos sugerencias:

a) Los proyectos educativos en salud, en especial en el campo de la odontología, deben tener presente la identificación de los valores y la importancia que los alumnos le asignan al momento de iniciar su formación en la educación superior, y

b) la declaración de intención formativa reflejada en el perfil de egreso debe contener y dar cuenta en el curriculum de las distintas acciones pedagógicas que se organizan para fortalecer, incentivar o desarrollar los valores declarados. 
Tabla $N^{\circ} 1$ : Prueba de Kruskal Wallis de los valores correspondientes a las primeras 5 jerarquías con la variable de agrupación "género".

\begin{tabular}{|c|c|c|c|c|c|c|}
\hline & Género & $\mathrm{N}$ & Rango promedio & Chi-cuadrado & $\mathrm{gl}$ & Sig. asintót. \\
\hline \multirow[t]{3}{*}{ Felicidad } & Masculino & 476 & 578,87 & 18,280 & 1 & ,000 \\
\hline & Femenino & 757 & 640,97 & & & \\
\hline & Total & 1233 & & & & \\
\hline \multirow[t]{3}{*}{ Responsabilidad } & Masculino & 476 & 565,59 & 26,642 & 1 & ,000 \\
\hline & Femenino & 757 & 649,33 & & & \\
\hline & Total & 1233 & & & & \\
\hline \multirow[t]{3}{*}{ Respeto } & Masculino & 476 & 573,04 & 18,203 & 1 & ,000 \\
\hline & Femenino & 757 & 644,64 & & & \\
\hline & Total & 1233 & & & & \\
\hline \multirow[t]{3}{*}{ Sinceridad } & Masculino & 478 & 567,22 & 26,300 & 1 & ,000 \\
\hline & Femenino & 758 & 650,84 & & & \\
\hline & Total & 1236 & & & & \\
\hline \multirow[t]{3}{*}{ Bienestar } & Masculino & 477 & 597,28 & 3,287 & 1 &, 070 \\
\hline & Femenino & 755 & 628,65 & & & \\
\hline & Total & 1232 & & & & \\
\hline
\end{tabular}

Tabla N²: Prueba de Kruskal Wallis de los valores jerarquizados en las últimas 5 preferencias con la variable de agrupación "género"

\begin{tabular}{|c|c|c|c|c|c|c|}
\hline & Género & $\mathrm{N}$ & Rango promedio & Chi-cuadrado & $\mathrm{gl}$ & Sig. asintót. \\
\hline Innovación & $\begin{array}{l}\text { Masculino } \\
\text { Femenino } \\
\text { Total }\end{array}$ & $\begin{array}{l}478 \\
756 \\
1234\end{array}$ & $\begin{array}{l}628,74 \\
610,40\end{array}$ & ,904 & 1 &, 0342 \\
\hline Liderazgo & $\begin{array}{l}\text { Masculino } \\
\text { Femenino } \\
\text { Total }\end{array}$ & $\begin{array}{l}477 \\
758 \\
1235\end{array}$ & $\begin{array}{l}640,81 \\
603,64\end{array}$ & 3,654 & 1 & 0,056 \\
\hline Civismo & $\begin{array}{l}\text { Masculino } \\
\text { Femenino } \\
\text { Total }\end{array}$ & $\begin{array}{l}476 \\
756 \\
1232\end{array}$ & $\begin{array}{l}601,73 \\
625,80\end{array}$ & 1,521 & 1 & 0,217 \\
\hline Participación & $\begin{array}{l}\text { Masculino } \\
\text { Femenino } \\
\text { Total }\end{array}$ & $\begin{array}{l}475 \\
754 \\
1229\end{array}$ & $\begin{array}{l}608,44 \\
619,13\end{array}$ & 0,305 & 1 & 0,581 \\
\hline Reconocimiento & $\begin{array}{l}\text { Masculino } \\
\text { Femenino } \\
\text { Total }\end{array}$ & $\begin{array}{l}478 \\
758 \\
1236\end{array}$ & $\begin{array}{l}608,06 \\
625,09\end{array}$ & 0,731 & 1 & 0,392 \\
\hline
\end{tabular}


Valores éticos y formación curricular en odontología - Luis A. Vicentela, Carmen Gloria Narváez, Manuel Velásquez

Tabla N³: prueba de Kruskal Wallis de los valores correspondientes a las primeras 5 jerarquías con la variable de agrupación "curso"

\begin{tabular}{|c|c|c|c|c|c|c|}
\hline & Curso & $\mathrm{N}$ & Rango promedio & Chi-cuadrado & $\mathrm{gl}$ & Sig. asintót. \\
\hline Felicidad & $\begin{array}{l}\text { Primer Año } \\
\text { Quinto Año } \\
\text { Total }\end{array}$ & $\begin{array}{l}711 \\
529 \\
1240\end{array}$ & $\begin{array}{l}627,12 \\
611,61\end{array}$ & 1,168 & 1 & 280 \\
\hline Responsabilidad & $\begin{array}{l}\text { Primer Año } \\
\text { Quinto Año } \\
\text { Total }\end{array}$ & $\begin{array}{l}710 \\
530 \\
1240\end{array}$ & $\begin{array}{l}626,40 \\
612,60\end{array}$ & ,743 & 1 & ,389 \\
\hline Respeto & $\begin{array}{l}\text { Primer Año } \\
\text { Quinto Año } \\
\text { Total }\end{array}$ & $\begin{array}{l}711 \\
529 \\
1240\end{array}$ & $\begin{array}{l}631,90 \\
605,18\end{array}$ & 2,600 & 1 &, 107 \\
\hline Sinceridad & $\begin{array}{l}\text { Primer Año } \\
\text { Quinto Año } \\
\text { Total }\end{array}$ & $\begin{array}{l}712 \\
531 \\
1243\end{array}$ & $\begin{array}{l}638,14 \\
600,35\end{array}$ & 5,501 & 1 & ,019 \\
\hline Bienestar & $\begin{array}{l}\text { Primer Año } \\
\text { Quinto Año } \\
\text { Total }\end{array}$ & $\begin{array}{l}710 \\
529 \\
1239\end{array}$ & $\begin{array}{l}631,57 \\
604,47\end{array}$ & 2,513 & 1 & ,113 \\
\hline
\end{tabular}

Tabla № 4: prueba de Kruskal Wallis de los valores jerarquizados en las últimas 5 preferencias con la variable de agrupación "curso"

\begin{tabular}{|c|c|c|c|c|c|c|}
\hline & Curso & $\mathrm{N}$ & Rango promedio & Chi-cuadrado & $\mathrm{gl}$ & Sig. asintót. \\
\hline Innovación & $\begin{array}{l}\text { Primer Año } \\
\text { Quinto Año } \\
\text { Total }\end{array}$ & $\begin{array}{l}710 \\
531 \\
1241\end{array}$ & $\begin{array}{l}652,24 \\
579,22\end{array}$ & 14,707 & 1 & ,000 \\
\hline Liderazgo & $\begin{array}{l}\text { Primer Año } \\
\text { Quinto Año } \\
\text { Total }\end{array}$ & $\begin{array}{l}711 \\
531 \\
1242\end{array}$ & $\begin{array}{l}654,07 \\
577,90\end{array}$ & 15,756 & 1 & ,000 \\
\hline Civismo & $\begin{array}{l}\text { Primer Año } \\
\text { Quinto Año } \\
\text { Total }\end{array}$ & $\begin{array}{l}708 \\
531 \\
1239\end{array}$ & $\begin{array}{l}636,52 \\
597,98\end{array}$ & 4,003 & 1 &, 045 \\
\hline Participación & $\begin{array}{l}\text { Primer Año } \\
\text { Quinto Año } \\
\text { Total }\end{array}$ & $\begin{array}{l}709 \\
527 \\
1236\end{array}$ & $\begin{array}{l}644,08 \\
584,08\end{array}$ & 9,836 & 1 & ,002 \\
\hline Reconocimiento & $\begin{array}{l}\text { Primer Año } \\
\text { Quinto Año } \\
\text { Total }\end{array}$ & $\begin{array}{l}712 \\
531 \\
1243 \\
\end{array}$ & $\begin{array}{l}645,78 \\
590,12\end{array}$ & 8,014 & 1 &, 005 \\
\hline
\end{tabular}


Tabla $\mathrm{N}^{\circ}$ 5: Valores presentes en los perfiles de egreso de las universidades encuestadas

\begin{tabular}{|l|c|c|c|c|c|c|c|c|}
\hline \multicolumn{7}{|c|}{ Valores } & \multicolumn{7}{c|}{ Universidades } \\
\hline & 1 & 2 & 3 & 4 & 5 & 6 & 7 & 8 \\
\hline Liderazgo & $\mathrm{X}$ & $\mathrm{X}$ & $\mathrm{X}$ & $\mathrm{X}$ & & & $\mathrm{X}$ & $\mathrm{X}$ \\
\hline Participación & $\mathrm{X}$ & & $\mathrm{X}$ & $\mathrm{X}$ & & $\mathrm{X}$ & $\mathrm{X}$ & $\mathrm{X}$ \\
\hline Cooperación & $\mathrm{X}$ & & $\mathrm{X}$ & $\mathrm{X}$ & & $\mathrm{X}$ & $\mathrm{X}$ & $\mathrm{X}$ \\
\hline Convivencia & $\mathrm{X}$ & & $\mathrm{X}$ & $\mathrm{X}$ & & $\mathrm{X}$ & $\mathrm{X}$ & $\mathrm{X}$ \\
\hline Seguridad & & $\mathrm{X}$ & & & & & & \\
\hline Innovación & & $\mathrm{X}$ & & & & & & $\mathrm{X}$ \\
\hline Responsabilidad & & & & & & $\mathrm{X}$ & & \\
\hline Autonomía & & & & & & $\mathrm{X}$ & & $\mathrm{X}$ \\
\hline Respeto & & & & & & & & $\mathrm{X}$ \\
\hline Sentido Crítico & & & & & & & & $\mathrm{X}$ \\
\hline
\end{tabular}

\section{Referencias}

1. Nash, DA. On ethics in the profession of dentistry and dental education. European Journal of Dental Education 2007; 11: 64-74.

2. Bridgman A, Collier A, Cunningham J. Teaching and assessing ethics and law in the dental curriculum. British Dental Journal 1999; 187(4): 217-219.

3. Torres Quintana M, Romo F. Bioética y ejercicio profesional de la odontología. Acta Bioethica 2006; 12(1): 65-74.

4. Zaror C, Vergara G. Ética en el currículo de las carreras de odontología. Acta Bioethica 2008; 14(2): 212-218.

5. Guerra RA. Bioética en la malla curricular de odontología. Acta Bioethica 2006; 12(1): 49-54.

6. Álvarez A. Valores de ética profesional en los estudiantes de la carrera de cirujano dentista. El significado del concepto “dentista competente" Revista ADM 2006; 64(4): 158-167.

7. Santos Guerra M. Una pretensión problemática: educar para los valores y preparar para la vida. Revista de Educación 2010; 351: 23-47.

8. Capote E, Villegas H, Brett M, Guada N, Capote J. Comparación de la jerarquía de valores entre los estudiantes de medicina y odontología del segundo año de la Universidad de Carabobo. Acta Odontológica Venezolana 2005; 44(3): 63-67.

9. García R, Ferrández M, Sales M, Moliner M. Elaboración de instrumentos de medida de las actitudes y opiniones del profesorado universitario hacia la ética profesional docente y su papel como trasmisor de valores. Revista electrónica de investigación y evaluación educativa 2006; 12(1): 129-149.

10. Lissabet Rivero JL. La utilización del método de evaluación de expertos en la valoración de los resultados de las investigaciones educativas. Manzanillo: Granma Ed; 1998.

11. Padilla MT. Técnicas e instrumentos para el diagnóstico y la evaluación educativa. Madrid: Editorial CCS; 2002.

Recibido: 17 de mayo de 2013

Aceptado: 25 de julio de 2013 